Mens

Revue d'histoire intellectuelle et culturelle

\title{
Le projet de Recours aux sources : réplique à Jacques Rouillard
}

\section{Éric Bédard}

Volume 13, numéro 1, automne 2012

S’approprier le passé des autres : les usages de l'histoire internationale au Québec avant la Révolution tranquille

URI : https://id.erudit.org/iderudit/1019701ar

DOI : https://doi.org/10.7202/1019701ar

Aller au sommaire du numéro

Éditeur(s)

Centre de recherche en civilisation canadienne-française

ISSN

1492-8647 (imprimé)

1927-9299 (numérique)

Découvrir la revue

Citer ce document

Bédard, É. (2012). Le projet de Recours aux sources : réplique à Jacques

Rouillard. Mens, 13(1), 113-122. https://doi.org/10.7202/1019701ar d'utilisation que vous pouvez consulter en ligne.

https://apropos.erudit.org/fr/usagers/politique-dutilisation/ 


\title{
Le projet de Recours aux sources : réplique à Jacques Rouillard
}

\author{
Éric Bédard \\ TÉLUQ, Université du Québec
}

Dans la dernière livraison de Mens, l'historien Jacques Rouillard consacre à Recours aux sources' ${ }^{1}$ une note critique que j'ai lue avec beaucoup d'intérêt' ${ }^{2}$. Je remercie mon ancien professeur de l'Université de Montréal d'avoir lu mon livre et d'avoir pris quelques heures de son temps pour écrire ce texte respectueux qui ne verse pas dans la polémique. L'éthique de la discussion dont témoigne sa note critique - et dont, plus jeune, je n'ai pas toujours fait preuve - ajoute à la grandeur de la vie académique et permet d'éclairer certains débats. Ces dialogues peuvent être instructifs et féconds dans la mesure où les thèses présentées ne déforment pas la pensée d'un auteur. Je remercie aussi Jacques Rouillard de considérer que Recours aux sources est à la fois une " tentative de faire le pont entre le présent et le passé " et une " réflexion originale " sur les "grands enjeux du débat public » (p. 145). Comme lui, j'ai parfois l'impression que «la république des historiens se ferme sur elle-même » (p. 145), que nous nous soucions trop peu du public et que nous nous tenons éloignés des débats politiques actuels. Les historiens devraient s'inspirer davantage du beau succès de La bataille de Londres, l'excellent ouvrage de Frédéric Bastien sur le rapatriement constitutionnel ${ }^{3}$.

1 Éric Bédard, Recours aux sources : essais sur notre rapport au passé, Montréal, Éditions du Boréal, 2011.

2 Jacques Rouillard, «Le retour aux sources selon Éric Bédard ", Mens : revue d'histoire intellectuelle et culturelle, vol. XII, n ${ }^{\circ} 2$ (printemps 2012), p. 145-163.

3 Frédéric Bastien, La bataille de Londres : dessous, secrets et coulisses du rapatriement constitutionnel, Montréal, Éditions du Boréal, 2013. 
Lorsqu'elle s'appuie sur des archives inédites plutôt que sur des concepts obscurs, qu'elle s'écrit dans une langue exempte d'un jargon indigeste et qu'elle traite d'un thème qui travaille la mémoire collective, l'histoire issue de l'université peut contribuer aux débats du présent.

Jacques Rouillard perçoit dans les essais de Recours aux sources un désenchantement par rapport à la société libérale et une " amertume " face à la liquidation du passé. Il se penche à peine sur la première idée sinon pour dire que bien des gens de ma génération la génération X - ont été marqués par l'" insécurité, le chômage, la désillusion » et le "déclin des idéologies dites progressistes» (p. 146). Presque tout son texte porte en fait sur la question du rapport au passé canadien-français. Ce que me reproche mon ancien professeur, c'est d' " adopte[r] sans broncher la représentation voulant que la Révolution tranquille marque, pour la société francophone, le passage brutal de la Grande Noirceur au monde moderne » (p. 147). Puisque je semble adhérer à cette idée reçue, il reprend des arguments bien étayés dans d'autres textes ${ }^{4}$ et propose une brève synthèse historiographique qui montre bien que le Québec d'avant 1960 n'était pas la société monolithique, rurale et cléricale célébrée dans les pages du Devoir d'Henri Bourassa ou dans celles de L'Action française de l'abbé Groulx. L'historien rappelle que, dans ce Canada français d'autrefois, il existait bel et bien une bourgeoisie francophone dynamique, favorable au développement industriel, dont les idées libérales disséminées dans un journal comme La Presse, le quotidien le plus populaire au début du $\mathrm{xx}^{\mathrm{e}}$ siècle, trouvaient un écho favorable au Parti libéral, bien installé au pouvoir de 1897 à 1936. Éminent spécialiste de l'histoire de la classe ouvrière, il rappelle également que le taux de syndicalisation des travailleurs canadiens-français se

${ }^{4}$ Jacques Rouillard, "À propos de Genèse des nations et cultures du Nouveau Monde de Gérard Bouchard : réplique à Éric Bédard ", Bulletin d'histoire politique, vol. $11, \mathrm{n}^{\circ} 1$ (automne 2002), p. 144-150; «La Révolution tranquille : rupture ou tournant?", Revue d'études canadiennes, vol. 2, no 4 (hiver 1998), p. 23-51. 
comparaît avantageusement à celui des travailleurs des autres provinces, que les unions internationales comptaient plus de membres que les syndicats catholiques. En somme, il me reproche de prendre au sérieux la thèse de la Grande Noirceur alors qu'il ne s'agirait que d'un mythe construit par une génération de sociologues inspirés par l'école de Chicago et popularisé par Pierre Elliott Trudeau dans La grève de l'amiante.

En tout respect pour mon estimé collègue, je dois lui dire qu'il enfonce des portes grandes ouvertes. Comment un historien de ma génération un tant soit peu à jour pourrait-il ignorer les faits incontestables qu'il rappelle à notre mémoire? Comment un lecteur même distrait du manuel de Linteau-Durocher-Robert, qu'à l'instar de tous les étudiants de ma promotion j'ai lu et relu, pourrait-il croire, encore aujourd'hui, que la modernité du Québec serait soudainement apparue le soir du 22 juin 1960 ? J'ai entrepris mes études supérieures à l'époque où les travaux de la génération de Jacques Rouillard servaient de référence. Tout au long de mes études doctorales, je me suis référé au livre incontournable de Fernande Roy sur les idées libérales des milieux d'affaires montréalais ${ }^{5}$. S'il a un jour la générosité de lire mes autres travaux, M. Rouillard constatera que je suis très loin de balayer les avancées de l'historiographie " moderniste " du revers de la main. Mes autres livres et mes interventions publiques montrent que ma perception du Québec d'avant 1960 tient tout à fait compte des travaux de sa génération ${ }^{6}$. Dans Les Réformistes, je

${ }^{5}$ Fernande Roy, Progrès, harmonie, liberté : le libéralisme des milieux d'affaires francophones à Montréal au tournant du siècle, Montréal, Éditions du Boréal, 1988.

6 Éric Bédard, "Quel mandat pour la Caisse? ", Le Devoir, 25 mars 2009; "La Révolution tranquille est-elle un bloc? ", Le Devoir, 22 juin 2010. Dans le cadre de la série "Dix journées qui ont fait le Québec " commanditée par la Grande Bibliothèque du Québec et la Fondation Lionel-Groulx, j’ai présenté une conférence sur la journée du 22 juin 1960. Dans la première partie de mon exposé, je propose un " état du Québec en 1960 " grandement inspiré des travaux des historiens modernistes. Voir le texte de ma conférence sur le site Web de la Fondation Lionel-Groulx : [http://www.fondationlionelgroulx.org/Le-22-juin1960-L-election-de-Jean.html]. 
crois avoir montré que le Canada français du milieu du XIx ${ }^{e}$ siècle n'était pas une société monolithique, que les idées libérales y occupaient une place centrale, que le développement économique préoccupait énormément nos élites, lesquelles étaient loin de souscrire au discours messianique des ultramontains ${ }^{7}$. Les travaux des modernistes occupent une place importante dans la quatrième partie de mon Histoire $d u$ Québec pour les nuls, consacrée à la modernisation du Québec ${ }^{8}$. Dès l'introduction, j'écris : "Trop de Québécois semblent croire que leur passé se résume à une désespérante "Grande Noirceur", sans grand intérêt pour le présent et pour l'avenir. Grave erreur ${ }^{9} . .$. " En fait, si le mythe de la Grande Noirceur m'irrite à ce point, c'est notamment parce qu'il fait l'impasse sur les recherches empiriques de la génération moderniste. Voilà ce que ne semble pas avoir saisi Jacques Rouillard. Dans les premières pages de Recours aux sources, j'évoque pourtant clairement ces considérations historiographiques ${ }^{10}$.

Nul doute dans mon esprit : les historiens de sa génération ont fait avancer nos connaissances. La Grande Noirceur à laquelle je me réfère dans Recours aux sources renvoie moins aux travaux des historiens modernistes qu'à la mémoire collective, ce fait de culture souvent difficile à conceptualiser mais néanmoins réel, et dont les historiens doivent tenir compte s'ils souhaitent rester pertinents. C'est qu'en dépit des recherches empiriques en histoire, le grand récit de la Révolution tranquille continue d'habiter l'imaginaire des Québécois et l'année 1960 reste une ligne de démarcation déterminante entre un avant et un après. Cette persistance du grand récit, l'historien Jocelyn Létourneau le constate chaque année lorsqu’il demande aux étudiants de première année du baccalauréat de lui raconter dans leurs propres mots l'histoire du Québec. Au fil du temps, il a compilé

7 Éric Bédard, Les Réformistes : une génération canadienne-française au milieu du XIX siècle, Montréal, Éditions du Boréal, 2009.

8 Éric Bédard, "La reconquête tranquille (1939-1967) ", Histoire du Québec pour les nuls, Paris, First, 2012, p. 205-263.

9 Ibid., p. 1.

10 Bédard, Recours aux sources, p. 12-14. 
près de 4000 récits qui, pour l'essentiel, reprennent la même trame héritée de la mémoire collective ${ }^{11}$. Ces récits sont à peine plus développés que ceux des élèves du secondaire qu'il avait étudiés durant les années $1980^{12}$. Devant une telle réalité, un constat s'impose : la mémoire collective et l'historiographie sont des mondes parallèles qui évoluent à des rythmes bien différents. Les chercheurs s'illusionnent s'ils croient que quelques livres et colloques arriveront à réformer ou à déconstruire cet imaginaire collectif. S'il est en grande partie faux empiriquement, le mythe de la Grande Noirceur continue d'exister, malgré les travaux des historiens modernistes. Le projet de Recours aux sources n'était pas de montrer que ce mythe n'a probablement aucun fondement - des historiens comme Jacques Rouillard s'y emploient depuis longtemps, l'affaire est classée -, mais d'illustrer certaines dérives engendrées par cette représentation du passé.

Les nations comme les individus sont libres de rompre avec leur passé et de se réinventer. Mais il y a généralement un prix à payer. En tournant le dos à la tradition, les révolutionnaires agissent souvent comme s'ils étaient les seuls représentants authentiques du Vrai et du Bien. Bénis par l'Histoire, ils s'emploient à transformer la société comme s'ils possédaient l'ultime vérité sur les fins dernières. Comme j'ai tenté de le montrer dans plusieurs essais de Recours aux sources, cette ingratitude a marqué de nombreux esprits durant les années 1960, au Québec et ailleurs dans le monde. On retrouve, à mon avis, cette arrogance chez un Pierre Angers, l'auteur de L'Activité éducative, le célèbre avis du Conseil supérieur de l'éducation, publié en 1971, qui annonçait l'avènement du "s'éduquant » et la mise en place de la dernière réforme pédagogique. On retrouve également ces prétentions démiurgiques chez les felquistes qui, croyant le peuple trop " aliéné ", plongèrent le Québec dans l'une des crises politiques les

11 Jocelyn Létourneau, "Les jeunes, l'histoire du Québec et son enseignement ", Le Québec entre son passé et ses passages, Montréal, Éditions Fides, 2010, p. 43-55.

12 Jocelyn Létourneau, "L'imaginaire historique des jeunes Québécois ", Revue d'histoire de l'Amérique française, vol. 41, n 4 (printemps 1988), p. 553-574. 
plus dramatiques de son histoire. Ce virus du déracinement, on le retrouve aussi chez Pierre Elliott Trudeau et ses émules, qui imposèrent aux Québécois le multiculturalisme chartiste qui invalida de nombreux articles de la loi 101, en plus de provoquer la crise des accommodements raisonnables. Ce syndrome de la table rase semble aussi avoir gagné les auteurs du dernier programme d'histoire au secondaire, qui oblitère des pans entiers de notre histoire politico-nationale. Le trait d'union entre ces dérives idéologiques et politiques, qui, chacune, font l'objet d'un chapitre de mon livre, c'est cette volonté commune de s'arracher à un passé obscurantiste, de rompre avec des institutions obsolètes et de créer de toutes pièces un "homme nouveau ", affranchi des pesanteurs de l'histoire, un Canadien-ouvert-sur-le-monde protégé par une Charte dans un cas, un Québécois-libéré-des-capitalistes-etdes-curés dans l'autre.

Le projet de Recours aux sources visait aussi à souligner que, malgré ce rapport vicié au passé, largement répandu chez nos élites, plusieurs Québécois restent attachés à une certaine continuité historique. C'est du moins ainsi que j'explique, dans mon premier chapitre, l'immense succès de la chanson Dégénérations du groupe Mes Aïeux en pleine crise des accommodements raisonnables. Le rapport plus empathique au passé et aux ancêtres a visiblement touché une corde sensible. Le succès populaire qu'a connu la série Duplessis, diffusée la première fois en 1978, est aussi un signe que les Québécois étaient prêts à reconsidérer le mythe de la Grande Noirceur. Le portrait du chef de l'Union nationale proposé par l'historien Jacques Lacoursière, le scénariste Denys Arcand et le réalisateur Mark Blandford, sans être complaisant, était plutôt sympathique à l'homme et à son régime. Dans un autre chapitre, je montre que le succès du Parti québécois tient en partie au rapport au passé de son fondateur. En effet, l'échec des pourparlers entre le Mouvement SouverainetéAssociation (MSA) et le Rassemblement pour l'indépendance nationale (RIN) ne tenait pas seulement à des querelles personnelles entre René Lévesque et Pierre Bourgault mais aussi, dans une certaine mesure, à des rapports différents au passé : le premier était soucieux 
de continuité alors que le second s'inscrivait dans la rupture la plus radicale et le discours de la décolonisation. Sans ce rapport plus empathique au passé, René Lévesque n'aurait pu rejoindre les nationalistes conservateurs qui continuaient de percevoir la souveraineté du Québec comme l'instrument par excellence de la survie ${ }^{13}$, non comme la promesse d'une société complètement nouvelle constituée de «bâtards » sans attaches ${ }^{14}$. Comme je l'ai signalé dans mon chapitre sur la « trudeauisation des esprits », l'adhésion des dirigeants péquistes et bloquistes au discours chartiste aux lendemains du référendum de 1995 leur aurait aliéné des franges importantes de l'électorat nationaliste ${ }^{15}$.

La cible de Recours aux sources est le rapport au temps de celles et de ceux qui se réclament du " progressisme ". Ceux-là semblent tenir pour acquis que l'histoire a un sens, qu'elle serait mue par des lois, qu'il ne faudrait, par conséquent, s'y intéresser que si les événements et les personnages étudiés permettent d'illustrer des changements, de dégager une trajectoire ou de décrire un avènement. L'histoire des progressistes est donc presque toujours téléologique, comme le furent d'ailleurs les grands travaux des historiens modernistes. Qu'ils aient été de sensibilité "libérale » ou "sociale-démocrate», les historiens modernistes ont surtout mis au jour les grands processus de la modernité occidentale : l'avènement des droits de la personne, la lutte pour la reconnaissance des Amérindiens ou des femmes, le développement du capitalisme, l'imposition des normes bourgeoises. En s'intéressant à ces grands processus économiques, sociaux et culturels, ils ont bel et bien montré que le Québec d'avant 1960 n'avait rien d'exceptionnel. Je ne leur reproche pas d'avoir fait cette

${ }^{13}$ Mathieu Bock-Côté, "La question du conservatisme ", Fin de cycle : aux origines du malaise politique québécois, Montréal, Éditions du Boréal, 2012, p. 121-149.

14 Je fais référence au paradigme du bâtard développé par Gérard Bouchard dans Genèse des nations et cultures du Nouveau Monde : essai d'histoire comparée, Montréal, Éditions du Boréal, 2000, p. 182.

15 Sur l'échec du Bloc, voir Bock-Côté, «Petite histoire d'une grande dérive : bilan du Bloc québécois ", Fin de cycle, p. 91-119. 
démonstration, encore moins d'avoir considérablement enrichi nos connaissances, mais de ne s'être intéressés qu'à ce qui annonçait notre époque. Face à ce métarécit d'une modernité glorieuse qui balaie tout sur son passage, j'ai toujours ressenti un malaise.

Je l'admets d'emblée, ma conception de l'histoire, exposée dans "L'héritage impossible " et l'épilogue de Recours aux sources, est conservatrice. Conservatrice en ce qui a trait aux objets de recherche d'une part. Je continue, en effet, de déplorer que nos grands départements universitaires négligent la recherche sur les événements marquants qui trônent au milieu de notre mémoire collective et qui continuent d'intéresser nos compatriotes : la fondation de Québec, la Conquête, les rébellions, les luttes constitutionnelles, la Révolution tranquille (en tant qu'événement) ${ }^{16}$. Les ouvrages les plus récents et les plus pertinents sur Champlain ou la guerre de Sept Ans ont été écrits par des Américains. On chercherait en vain dans les départements d'histoire de nos universités francophones, un vrai spécialiste des patriotes. Aucune des grandes biographies sur Trudeau, Lévesque ou les acteurs clés de la Révolution tranquille n’ont été écrites par des historiens universitaires. Mobilisée par l'étude des grands processus de la modernité, la génération de Jacques Rouillard a délaissé une histoire plus traditionnelle, moins captive des concepts à la mode en sciences sociales, centrée sur des événements particuliers ou des personnages politiques marquants; une histoire qui tient compte de la contingence et qui chercherait moins à valider une théorie - une théorie sur le libéralisme, par exemple ${ }^{17}$ - qu’à proposer une mise en

16 Là-dessus, voir L’histoire nationale négligée, mon enquête commanditée par la Fondation Lionel-Groulx, parue en octobre 2011 et réalisée avec la collaboration de Myriam D’Arcy, sur le site Web de la Fondation Lionel-Groulx : [http://www. fondationlionelgroulx.org/IMG/pdf/l-histoire-nationale-negligee.pdf]. Dans la postface de son ouvrage, Frédéric Bastien raconte qu'il n'a reçu aucun soutien d'une institution universitaire ni aucune subvention du Conseil de recherches en sciences humaines (Bastien, La bataille de Londres, p. 451-458).

17 Deux exemples de cette approche par "validation ", plus proche de la sociologie ou de la science politique que d'une histoire sensible à la contingence : Jean-Marie Fecteau, La liberté du pauvre : crime et pauvreté au XIXe siècle québécois, Montréal, 
récit du passé induite par des archives patiemment dépouillées; une histoire qui permettrait de répondre à une question toute simple que se pose le grand public lorsqu'il daigne ouvrir un livre d'histoire : "Mais que s'est-il donc passé ${ }^{18}$ ? " Je souhaite que les étudiants intéressés par l'histoire politico-nationale puissent avoir accès à des chercheurs reconnus et disposer d'un environnement intellectuel stimulant. Une certaine gauche universitaire, très présente en histoire, aime bien donner des leçons de " diversité ". Le temps ne serait-il pas venu de prêcher par l'exemple?

Mon conservatisme d'historien est surtout philosophique. Le titre de mon livre n'est pas qu'un jeu de mots, c'est un programme éthique. J'aime trop mon époque et les immenses possibilités qu'elle offre pour rêver d'un « retour » en arrière, mais je n'oublie jamais que ce monde dont j'hérite est le fruit du travail patient des générations qui me précèdent. Mon rapport au passé est en effet celui d'un " héritier reconnaissant ", non d'un justicier qui distribuerait aux uns des certificats de bonne conduite et aux autres des sauf-conduits pour le musée des horreurs, selon qu'ils aient ou non annoncé les valeurs bienfaisantes de notre époque. Se tourner vers le passé, c'est se rappeler que nous ne nous sommes pas faits tout seuls, que d'autres, avant nous, ont préparé le terrain à notre venue. S’il faut aussi explorer le passé, c'est par prudence ${ }^{19}$. L'histoire devrait normalement nous immuniser contre les discours de la " table rase " professés par tous ces prophètes qui croient avoir trouvé le sens ultime de l'Histoire celle qui s'écrit avec une grande "hache ", selon le mot de Georges

VLB éditeur, 2004 ; Michel Ducharme, Le concept de liberté au Canada à l'époque des révolutions atlantiques : 1776-1838, Montréal, McGill-Queen's University Press, 2010.

18 Parmi les ouvrages qui ont inspiré cet attachement décomplexé à une histoire plus traditionnelle, voir Keith Windschuttle, The Killing of History: How Literary Critics and Social Theorists Are Murdering Our Past, San Francisco, Encounter Books, 2000; G. R. Elton, Return to Essentials: Some Reflections on the Present State of Historical Study, Cambridge, Cambridge University Press, 1991.

19 Là-dessus, voir Gordon Wood, The Purpose of the Past: Reflections on the Uses of History, New York, Penguin Press, 2008. 
Perec. Depuis Hérodote, nous savons que l'histoire n'est pas écrite d'avance, que son sens ultime nous échappe. C'est donc dire que nous conservons une part de liberté, que nous avons la possibilité d'en changer le cours par nos actions, nos écrits, notre travail. Ce rapport conservateur au passé, fait d'empathie pour les devanciers et de prudence politique, a souvent guidé nos élites canadiennesfrançaises, pour le meilleur et pour le pire. Nous lui devons peut-être notre survie comme peuple. 